\title{
Sciendo
}

Int. J. of Applied Mechanics and Engineering, 2020, vol.25, No.2, pp.176-191

DOI: $10.2478 /$ ijame-2020-0028

\section{INVESTIGATION OF VELOCITY PROFILE IN TIME DEPENDENT BOUNDARY LAYER FLOW OF A MODIFIED POWER-LAW FLUID OF FOURTH GRADE}

\author{
M. YÜRÜSOY \\ Department of Mechanical Engineering \\ Afyon Kocatepe University 03200 \\ Afyonkarahisar, TURKEY \\ E-mail: yurusoy@aku.edu.tr
}

\begin{abstract}
This paper deals with the investigation of time dependent boundary layer flow of a modified power-law fluid of fourth grade on a stretched surface with an injection or suction boundary condition. The fluid model is a mixture of fourth grade and power-law fluids in which the fluid may display shear thickening, shear thinning or normal stress textures. By using the scaling and translation transformations which is a type of Lie Group transformation, time dependent boundary layer equations are reduced into two alternative ordinary differential equations systems (ODEs) with boundary conditions. During this reduction, special Lie Group transformations are used for translation, scaling and combined transformation. Numerical solutions have been carried out for the ordinary differential equations for various fluids and boundary condition parameters. As a result of numerical analysis, it is observed that the boundary layer thickness decreases as the power-law index value increases. It was also observed that for the fourth-grade fluid parameter, as the parameter increases, the boundary layer thickness decreases while the velocity in the $y$ direction increases.
\end{abstract}

Key words: boundary layer equations, power-law fluids, fourth grade fluids.

\section{Introduction}

Many models have been developed to determine non-Newtonian behavior of fluids. The most important of these are of differential type, power-law and rate type models. Along with many flow problems, boundary layer theory has been successfully applied to these models. Both non-Newtonian fluids and the boundary layer theory have many industrial applications, such as the production of paper in the manufacturing of materials by an extrusion process, spinning of metal, blowing of glass, and so on. For the solution of mathematical models for the flow of non-Newtonian fluids, the Lie Group theory is widely used. Applications of Lie Groups to differential equations in search of exact solutions have been well-established for over a century. The method has been applied to a wide range of problems in fluid mechanics, elasticity, quantum mechanics, heat transfer, differential geometry and astronomy, etc. In the method of group theory, partial differential equations are generally reduced to ordinary differential equations by finding the symmetries of the equations. The algebra involved in the analysis becomes quite complicated, especially, when the number of variables or the order of differential equation increases. However, the existing algorithms run in a straightforward manner and cannot handle cases when there are arbitrary functions involved in the equations. For some specific forms of these arbitrary functions, the symmetries of the differential equations are richer and determination of these specific forms turns out to be a group classification problem. For problems having arbitrary functions, several independent and dependent variables, or higher orders of differentiation, one practical approach might be to use special group transformations such as scaling, translation, spiral, Galilean, etc. to produce quick results. As an example, in boundary layer problems, we know that scaling, translation and spiral group of transformations are the most common ones yielding physical solutions [1-16]. Hence, for a new boundary layer problem, the initial 
attempt might be to try these special transformations before applying the general Lie group analysis. Motivated by the previous work on boundary layers of non-Newtonian fluids [1-16], we propose here a transformation which includes scaling, translation and spiral group of transformations as its special cases. Like the other special transformations, this transformation would be useful especially for an applied oriented researcher who is seeking a physical solution of his problem without involving deeply into the theory. We apply the transformation to time dependent boundary layer equations for a modified power-law fluid of fourth grade. By using a few special Lie Group transformations, the time dependent boundary layer equation system is reduced into an ordinary differential equation system. Moving surface or stretched sheet and suction or injection surface boundary condition are considered, and a Runge-Kutta shooting method is used to solve the equations.

\section{Equation of motion}

A non-Newtonian fluid model, power-law, second, third and fourth grade fluids are largely used by investigators. The basic stress equation for the modified power-law fluid of fourth grade is as follows

$$
\begin{aligned}
& \mathrm{T}=-p I+\mu \Pi^{m / 2} A_{1}+\alpha_{1} A_{2}+\alpha_{2} A_{1}^{2}+\beta_{1} A_{3}+\beta_{2}\left(A_{2} A_{1}+A_{1} A_{2}\right)+\beta_{3}\left(\operatorname{tr}\left(A_{1}^{2}\right)\right) A_{1}+\gamma_{1} A_{4}+ \\
& +\gamma_{2}\left(A_{3} A_{1}+A_{1} A_{3}\right)+\gamma_{3} A_{2}^{2}+\gamma_{4}\left(A_{2} A_{1}^{2}+A_{1}^{2} A_{2}\right)+\gamma_{5}\left(\operatorname{tr}\left(A_{2}\right)\right) A_{2}+\gamma_{6}\left(\operatorname{tr}\left(A_{2}\right)\right) A_{1}^{2}+ \\
& +\left(\gamma_{7}\left(\operatorname{tr}\left(A_{3}\right)\right)+\gamma_{8} \operatorname{tr}\left(A_{2} A_{1}\right)\right) A_{1}
\end{aligned}
$$

where $\mathbf{T}$ is the Cauchy stress tensor, $\boldsymbol{I}$ is identity tensor, $p$ is the pressure, $\mu$ is the dynamic viscosity, $\alpha_{l}$ and $\alpha_{2}$ are second grade fluid material constants, $\beta_{1}, \beta_{2}, \beta_{3}$ are third grade fluids material constants. $\gamma_{i}=1,2,3,4$, 5, 6, 7, 8 are fourth grade fluid material constants, $m$ is the power-law fluid index. $\boldsymbol{A}_{1}, \boldsymbol{A}_{2}, \boldsymbol{A}_{3}$ and $\boldsymbol{A}_{4}$ are Rivlin-Ericksen tensors defined by

$$
\begin{aligned}
& A_{n}=\dot{A}_{n-1}+A_{n-1} L+L^{T} A_{n-1} \quad n>1, \\
& A_{1}=A_{1} L+L^{T} A_{1}
\end{aligned}
$$

where $\boldsymbol{L}=(\nabla \boldsymbol{V}), \boldsymbol{V}=(u, v)$ is the velocity vector and $(\cdot)$ is the material time derivative. For this model, if $m=0$, the equations reduce to those of fourth grade fluids, whereas, if $\alpha_{l}=\alpha_{2}=0, \beta_{l}=\beta_{2}=\beta_{3}=0, \gamma_{i}=0$ and $m=0$ then the fluid is a Newtonian fluid. The fluid is known to show shear thickening behavior when $m>0$, shear thinning behavior when $m<0$.

Mass conservation and momentum equations are

$$
\begin{aligned}
& \operatorname{div}(\boldsymbol{V})=0, \\
& \rho \frac{d \boldsymbol{V}}{d t}=\operatorname{div}(\boldsymbol{T})+\rho b .
\end{aligned}
$$

To derive the unsteady boundary layer equations, we will set a coordinate system in which $x$ is the coordinate along the boundary and $y$ is the coordinate normal to it. Substituting Eqs (2.1) and (2.2) into Eqs (2.3) and (2.4), and under the usual boundary layer, the assumption that one finally obtains is the continuity and unsteady boundary layer equations for the modified fourth grade fluid. 


$$
\begin{aligned}
& u_{x}+v_{y}=0 \\
& u_{t}+u u_{x}+v u_{y}=\varepsilon(m+1)\left(\left|u_{y}\right|\right)^{m} u_{y y}+\widehat{\varepsilon}_{1}\left[u_{x} u_{y y}-u_{y} u_{x y}+v u_{y y y}+u u_{x y y}+u_{t y y}\right]+ \\
& +6 \widehat{\beta}\left(u_{y}\right)^{2} u_{y y}+8 \widehat{\gamma}\left(u_{y}\right)^{3} u_{x y}+\widehat{\gamma}_{1}\left[\left(u_{y}\right)^{3} u_{x y}+2 u_{y} u_{y y}{ }^{2} v+u_{y}{ }^{2} v u_{y y y}+\left(u_{y}\right)^{2} u u_{x y y}+\right. \\
& \left.-u u_{y y}\left(u_{y}\right)^{2}+2 u_{y y} u_{x y} u_{y} u+u_{t y y}\left(u_{y}\right)^{2}+2 u_{y} u_{y y} u_{t y}\right]+V_{t}+V V_{x}
\end{aligned}
$$

where $V(x, t)$ is the $x$ component of the velocity outside the boundary layer. The dimensionless parameters are defined as

$$
\begin{aligned}
& \varepsilon=\frac{v}{V L}\left(\frac{V}{L}\right)^{m}, \quad \widehat{\varepsilon}_{1}=\frac{\alpha_{1}}{\rho L^{2}}, \quad \widehat{\beta}=\frac{V\left(\beta_{2}+\beta_{3}\right)}{\rho L^{3}}, \quad \hat{\gamma}=\frac{\gamma_{6} V^{2}}{\rho L^{4}}, \\
& \widehat{\gamma}_{1}=\frac{2\left(3 \gamma_{2}+\gamma_{3}+\gamma_{4}+\gamma_{5}+3 \gamma_{7}+\gamma_{8}\right) V^{2}}{\rho L^{4}}, \quad v=\frac{\mu}{\rho}
\end{aligned}
$$

where $v$ is kinematic viscosity. According to the usual assumptions in the boundary layer theory, $x$ and $u$ is $O(1)$ and $v$ is of order $\delta(O(\delta))$. The dimensionless parameters must be as follows

$$
\varepsilon=\delta^{m+2}, \quad \widehat{\varepsilon}_{1}=\varepsilon_{1} \delta^{2}, \quad \widehat{\beta}=\beta \delta^{4}, \quad \widehat{\gamma}=\gamma \delta^{4}, \quad \widehat{\gamma}_{1}=\gamma_{1} \delta^{4}
$$
as follows

Equation (2.7) is used for unsteady boundary layer Eqs (2.4) and (2.5). New equations are obtained

$$
\begin{aligned}
& u_{x}+v_{y}=0 \\
& u_{t}+u u_{x}+v u_{y}=\varepsilon(m+1)\left(\left|u_{y}\right|\right)^{m} u_{y y}+\varepsilon_{1}\left[u_{x} u_{y y}-u_{y} u_{x y}+v u_{y y y}+u u_{x y y}+u_{t y y}\right]+ \\
& +6 \beta\left(u_{y}\right)^{2} u_{y y}+8 \gamma\left(u_{y}\right)^{3} u_{x y}+\gamma_{1}\left[\left(u_{y}\right)^{3} u_{x y}+2 u_{y} u_{y y}{ }^{2} v+u_{y}{ }^{2} v u_{y y y}+\left(u_{y}\right)^{2} u u_{x y y}+\right. \\
& \left.-u u_{y y}\left(u_{y}\right)^{2}+2 u_{y y} u_{x y} u_{y} u+u_{t y y}\left(u_{y}\right)^{2}+2 u_{y} u_{y y} u_{t y}\right]+V_{t}+V V_{x} .
\end{aligned}
$$

In this section, the time dependent boundary layer equations for the modified fourth order fluids were obtained.

\section{Symmetry analysis of boundary layers equations}

If Eq.(2.1) is also taken as $\varepsilon_{1}=0, \beta=0, \gamma_{1}=0$, the equations obtained are written as follows

$$
\begin{aligned}
& \frac{\partial u}{\partial x}+\frac{\partial v}{\partial y}=0 \\
& \frac{\partial u}{\partial t}+u \frac{\partial u}{\partial x}+v \frac{\partial u}{\partial y}=(m+1)\left(\left|\frac{\partial u}{\partial y}\right|\right)^{m} \frac{\partial^{2} u}{\partial y^{2}}+8 \gamma\left(\frac{\partial u}{\partial y}\right)^{3} \frac{\partial^{2} u}{\partial x \partial y}+\frac{\partial V}{\partial t}+V \frac{\partial V}{\partial x}
\end{aligned}
$$


In Eq.(2.9), $\varepsilon_{l}, \beta, \gamma$ and $\gamma_{l}$ refer to the second-grade fluid parameter, the third-grade fluid parameter, the fourth-grade fluid parameter and fluid effects, respectively. In the equation of motion, there are two fourth-order fluid parameters, solutions for the other parameter, that is $(\gamma)$, are produced by selecting $\gamma_{1}=0$.

Here, the second, third and fourth grade parameter terms refer to the grade of a non-Newtonian fluid. As the grade of a non-Newtonian fluid increases, the non-Newtonian character also increases. Equation (3.1) defines the continuity equation, while Eq.(3.2) refers to the boundary layer equation consisting of a combination of power-law and fourth-order fluid model for unsteady flow. Equation (3.3) describes the boundary conditions of the movement. Taking into account an unsteady boundary layer flow on a moving surface or stretched sheet surface with suction or injection, we can write the general boundary conditions as

$$
u(x, t, 0)=C(x, t), v(x, t, 0)=\mp D(x, t), u(x, t, \infty)=V(x, t) .
$$

Two type of reductions for Eqs (3.1) and (3.2) with boundary condition Eqs (3.3) will be presented. In the first, the reduction scaling transformation and in the other, translation transformation will be used.

\subsection{Scaling transformation}

We will present that the boundary layer equations admit scaling symmetry and reduce the equations to two independent partial differential equations in a more systematic way. Consider now the following scaling transformation

$$
\hat{t}=\lambda^{\varepsilon a_{1}} t, \quad \hat{x}=\lambda^{\varepsilon a_{2}} x, \quad \hat{y}=\lambda^{\varepsilon a_{3}} y, \quad \hat{u}=\lambda^{\varepsilon a_{4}} u, \quad \hat{v}=\lambda^{\varepsilon a_{5}} v, \quad \hat{V}=\lambda^{\varepsilon a_{6}} V
$$

where $\varepsilon$ is the transformation parameter. Substituting (3.4) into Eqs (3.2) and (3.3), dividing the equation by coefficient of the leading term, we have

$$
\begin{aligned}
& \frac{\partial u}{\partial x}+\frac{\partial v}{\partial y} e^{\varepsilon\left(a_{4}+a_{3}-a_{5}-a_{2}\right)}=0 \\
& \frac{\partial \hat{u}}{\partial \hat{t}}+\hat{u} \frac{\partial \hat{u}}{\partial \hat{x}} e^{\varepsilon\left(a_{2}-a_{1}-a_{4}\right)}+\hat{v} \frac{\partial \hat{u}}{\partial \hat{y}} e^{\varepsilon\left(a_{3}-a_{1}-a_{5}\right)}=(m+1)\left(\left|\frac{\partial \hat{u}}{\partial \hat{y}}\right|\right)^{m} \frac{\partial^{2} \hat{u}}{\partial \hat{y}^{2}} e^{\varepsilon\left[m\left(a_{3}-a_{4}\right)+2 a_{3}-a_{1}\right]}+ \\
& +8 \gamma\left(\frac{\partial \hat{u}}{\partial \hat{y}}\right)^{3} \frac{\partial^{2} \hat{u}}{\partial \hat{x} \partial \hat{y}} e^{\varepsilon\left(4 a_{3}+a_{2}-a_{1}-3 a_{4}\right)}+\frac{\partial \hat{V}}{\partial \hat{t}} e^{\varepsilon\left(a_{4}-a_{6}\right)}+\hat{V} \frac{\partial \hat{V}}{\partial \hat{x}} e^{\varepsilon\left(a_{2}+a_{4}-a_{1}-2 a_{6}\right)} .
\end{aligned}
$$

Comparing the transformed Eqs (3.5) and (3.6) with the original equation, we write the invariance condition

$$
\begin{aligned}
& a_{4}+a_{3}-a_{5}-a_{2}=0, \quad a_{2}-a_{1}-a_{4}=0, \quad a_{3}-a_{1}-a_{5}=0, \quad m\left(a_{3}-a_{4}\right)+2 a_{3}-a_{1}=0, \\
& 4 a_{3}+a_{2}-a_{1}-3 a_{4}=0, \quad a_{4}-a_{6}=0, \quad a_{2}+a_{4}-a_{1}-2 a_{6}=0 .
\end{aligned}
$$

Solving Eqs (3.7), we find as follows

$$
a_{1}=a_{3}(2-m), \quad a_{2}=a_{3}(4-m), \quad a_{4}=2 a_{3}, \quad a_{5}=a_{3}(m-1), \quad a_{6}=2 a_{3} .
$$

We set $a_{3}=1$ in Eq.(3.8). The equations for similarity transformations are 


$$
\frac{d t}{(2-m) t}=\frac{d x}{(4-m) x}=\frac{d y}{y}=\frac{d u}{2 u}=\frac{d v}{(m-1) v}=\frac{d V}{2 V} .
$$

Using the method of characteristics for solving (3.9), we have

$$
\zeta=\frac{x}{\frac{m-4}{t^{m-2}}}, \quad \theta=\frac{y}{\frac{1}{t^{2-m}}}, \quad u=t^{\frac{2}{2-m}} K(\zeta, \theta), \quad v=t^{\frac{m-1}{m-2}} N(\zeta, \theta), \quad V=t^{\frac{2}{2-m}} R(\zeta) .
$$

We assume the arbitrary function $C(x, t)$ and $D(x, t)$ in Eq.(3.3) as follows

$$
C(x, t)=t^{\frac{2}{2-m}} C(\zeta), \quad D(x, t)=\frac{D(\zeta)}{t^{\frac{m-1}{m-2}}} .
$$

Substituting (3.10) and (3.11) into the Eqs (3.1), (3.2) and (3.3), we reduce a three-independentvariable partial differential system to a two-independent-variable partial differential system

$$
\begin{aligned}
& \frac{\partial K}{\partial \zeta}+\frac{\partial N}{\partial \theta}=0 \\
& \frac{2}{2-m} K-\frac{m-4}{m-2} \frac{\partial K}{\partial \zeta} \zeta+\frac{1}{m-2} \frac{\partial K}{\partial \theta} \theta+K \frac{\partial K}{\partial \zeta}+N \frac{\partial K}{\partial \theta}=(m+1)\left(\left|\frac{\partial K}{\partial \theta}\right|\right)^{m} \frac{\partial^{2} K}{\partial \theta^{2}}+ \\
& +8 \gamma\left(\frac{\partial K}{\partial \theta}\right)^{3} \frac{\partial^{2} K}{\partial \zeta \partial \theta}+\frac{2}{2-m} R-\frac{m-4}{m-2} R^{\prime} \zeta+R R^{\prime} \\
& K(\zeta, 0)=C(\zeta), \quad N(\zeta, 0)=\mp D(\zeta), \quad K(\zeta, \infty)=R(\zeta) .
\end{aligned}
$$

\subsection{Translation transformation}

Consider now the following translation transformation for Eqs (3.1) and (3.2) is

$$
\begin{aligned}
& \hat{t}=t+\varepsilon a_{1}, \\
& \hat{x}=x+\varepsilon a_{2}, \\
& \hat{y}=y+\varepsilon a_{3}, \\
& \hat{u}=u+\varepsilon a_{4}, \\
& \hat{v}=v+\varepsilon a_{5}, \\
& \hat{V}=V+\varepsilon a_{6} .
\end{aligned}
$$


Substituting (3.15) into Eqs (3.2) and (3.3), we obtain $a_{4}=a_{5}=a_{6}=0$. There are no restrictions on the parameters $a_{1}, a_{2}$ and $a_{3}$. For a special condition, we set $a_{3}=0$. We obtain

$$
\frac{d t}{a_{1}}=\frac{d x}{a_{2}}=\frac{d y}{0}=\frac{d u}{0}=\frac{d v}{0}=\frac{d V}{0} .
$$

Using the method of characteristics for solving Eq.(25), we have

$$
\phi=n x-t, \quad \tau=y, \quad u=F(\phi, \tau), \quad v=Q(\phi, \tau), \quad V=R(\phi)
$$

where $n=a_{1} / a_{2}$, substituting (3.17) into the Eqs (3.1), (3.2) and (3.3), we reduce a three-independent-variable partial differential system into a two-independent-variable partial differential system for the second time

$$
\begin{aligned}
& n \frac{\partial F}{\partial \phi}+\frac{\partial Q}{\partial \tau}=0, \\
& -\frac{\partial F}{\partial \phi}+n F \frac{\partial F}{\partial \phi}+Q \frac{\partial F}{\partial \tau}=(m+1)\left(\left|\frac{\partial F}{\partial \tau}\right|\right)^{m} \frac{\partial^{2} F}{\partial \tau^{2}}+8 \gamma n\left(\frac{\partial F}{\partial \tau}\right)^{3} \frac{\partial^{2} F}{\partial \phi \partial \tau}-R^{\prime}+n R R^{\prime}, \\
& F(\phi, 0)=C(\phi), \quad Q(\phi, 0)=\mp D(\phi), \quad F(\phi, \infty)=R(\phi) .
\end{aligned}
$$

Thus, two different boundary layer equations having the two independent variable partial differential equations were obtained in this section.

\section{Further symmetry analysis}

Two independent variable partial differential equations obtained by using similarity transformations in the previous section will be reduced to ordinary differential equations in this section.

\subsection{Translation transformation}

Firstly, Eqs (3.12) and (3.13) will be discussed. The following translation transformation is considered for the equations

$$
\hat{\zeta}=\zeta+\varepsilon a_{1}, \quad \hat{\theta}=\theta+\varepsilon a_{2}, \quad \hat{K}=K+\varepsilon a_{3}, \quad \hat{N}=N+\varepsilon a_{4}, \quad \hat{R}=R+\varepsilon a_{5} .
$$

Substituting (4.1) into Eqs (3.12) and (3.13), we obtain

$$
a_{3}=\frac{m-4}{m-2} a_{1}, \quad a_{5}=\frac{m-4}{m-2} a_{1}, \quad a_{4}=\frac{1}{2-m} a_{2},
$$

if $a l=a_{2}=1$ is selected, we obtain

$$
\frac{d \zeta}{l}=\frac{d \theta}{1}=\frac{d K}{\frac{m-4}{m-2}}=\frac{d N}{\frac{1}{2-m}}=\frac{d R}{\frac{m-4}{m-2}},
$$


when the Eq.(4.3) is solved, we have

$$
\lambda=\theta-\zeta, \quad K=\frac{m-4}{m-2} \zeta+P(\lambda), \quad N=\frac{1}{2-m} \theta+G(\lambda), \quad R=\frac{m-4}{m-2} \zeta+c_{2} .
$$

We assume the arbitrary function $C(\zeta)$ and $D(\zeta)$ in Eq.(3.1423) as follows

$$
C=\frac{m-4}{m-2} \zeta+c_{1}, \quad D=\mp c .
$$

Substituting (4.4) and (4.5) into the Eqs (3.12), (3.13) and (3.14), we reduce a two-independentvariable partial differential system to an ordinary differential equations system

$$
\begin{aligned}
& \frac{m-5}{m-2}-P^{\prime}+G^{\prime}=0, \\
& \frac{m-6}{m-2} P+P^{\prime}(G-P)=(m+1)\left(\left|P^{\prime}\right|\right)^{m} P^{\prime \prime}-8 \gamma\left(P^{\prime}\right)^{3} P^{\prime \prime}+\frac{m-6}{m-2} c_{2}, \\
& P(0)=c_{1}, \quad G(0)=\mp c, \quad P(\infty)=c_{2} .
\end{aligned}
$$

\subsection{A combined transformation}

Consider now the following special Lie Group transformation

$$
\begin{aligned}
& \hat{\phi}=e^{\varepsilon a_{1}} \phi+\varepsilon b_{1}, \\
& \hat{\tau}=e^{\varepsilon a_{2}} \tau+\varepsilon b_{2}, \\
& \hat{F}=e^{\varepsilon a_{3}} F+\varepsilon b_{3}, \\
& \hat{Q}=e^{\varepsilon a_{4}} Q+\varepsilon b_{4}, \\
& \hat{R}=e^{\varepsilon a_{5}} R+\varepsilon b_{5}
\end{aligned}
$$

$b_{1}=b_{2}=b_{3}=b_{4}=b_{5}=0$ corresponds to the scaling transformation, $a_{1}=a_{2}=a_{3}=a_{4}=a_{5}=0$ corresponds to the translation transformation and $a_{1}=b_{2}=b_{3}=b_{4}=b_{5}=0$ corresponds to the spiral group transformation. Substituting Eqs (4.8) into Eqs (3.12) and (3.13) and dividing the equation by the coefficient of the leading term, we obtain

$$
a_{1}=(4-m) a_{2}, \quad a_{4}=(m-1) a_{2}, \quad a_{3}=2 a_{2}, \quad b_{3}=-\frac{2 a_{2}}{n}, \quad a_{5}=a_{3}, \quad b_{5}=-\frac{2 a_{2}}{n},
$$

if $b_{1}=b_{2}=0$ and $a_{2}=1$ are selected, we obtain 


$$
\frac{d \phi}{(4-m) \phi}=\frac{d \tau}{\tau}=\frac{d F}{\frac{2(n F-1)}{n}}=\frac{d Q}{(m-1) Q}=\frac{d R}{\frac{2(n R-1)}{n}}
$$

when the Eq.(32) is solved, we have

$$
\mu=\frac{\tau}{\phi^{\frac{1}{4-m}}}, \quad F=\frac{1}{2 n}\left(\phi^{\frac{2}{4-m}} S(\mu)+2\right), \quad Q=\phi^{\frac{m-1}{m-2}} K(\mu), \quad F=\frac{1}{2 n}\left(\phi^{\frac{2}{4-m}} c_{2}+2\right) .
$$

We assume the arbitrary function $C(\phi)$ and $D(\phi)$ in Eq.(3.20) as follows

$$
C=\frac{1}{2 n}\left(\phi^{\frac{2}{4-m}} c_{1}+2\right), \quad D=\frac{1}{2 n}\left(\phi^{\frac{2}{4-m}} c+2\right) .
$$

Substituting Eqs (4.11) and (4.12) into the Eqs (3.18), (3.19) and (3.20), we reduce a two independent variable partial differential system to an ordinary differential equations system

$$
\begin{aligned}
& \frac{1}{2} S^{\prime} \mu-S+(m-4) K^{\prime}=0 \\
& \frac{1}{4 n(m-4)} S S^{\prime} \mu-\frac{1}{2 n(m-4)} S^{2}+\frac{1}{2 n} K S^{\prime}=\frac{(m+1)}{n 2^{m+1}}\left(\left|S^{\prime}\right|\right)^{m} S^{\prime \prime}+ \\
& +\frac{\gamma}{2 n^{3}(m-4)}\left(\left(S^{\prime}\right)^{3} S^{\prime \prime} \mu-\left(S^{\prime}\right)^{4}\right)-\frac{c_{2}^{2}}{2 n(m-4)}, \\
& S(0)=c_{1}, \quad K(0)=\mp c, \quad S(\infty)=c_{2} .
\end{aligned}
$$

In this section, time dependent boundary layer equations have been reduced to the form of ordinary differential equations with two alternative approaches.

The shear stress expression for the fourth grade and power-law fluid model is as follows.

$$
\begin{aligned}
& \tau_{x y}=\left(\left|\frac{\partial u}{\partial y}\right|^{m} \frac{\partial u}{\partial y}+2\left(3\left(\gamma_{2}+\gamma_{7}\right)+\gamma_{8}\right)\left(\frac{\partial u}{\partial y}\right)^{2}\left(v \frac{\partial^{2} u}{\partial y^{2}}+u \frac{\partial^{2} u}{\partial x \partial y}\right)+\right. \\
& \left.+2\left(\gamma_{3}+\gamma_{4}+\gamma_{5}\right)\left(\frac{\partial u}{\partial y}\right)^{2}\left(v \frac{\partial^{2} u}{\partial y^{2}}+u \frac{\partial^{2} u}{\partial x \partial y}+2 \frac{\partial u}{\partial y} \frac{\partial u}{\partial x}\right)\right) .
\end{aligned}
$$

As can be seen from the equation above, the $\gamma_{6}$ parameter has no effect on shear stress.

\section{Numerical results}

By using a Runge-Kutta shooting technique, Eqs (4.13) and (4.14) are integrated, subjected to the boundary conditions (4.15). In Fig. $1, S$ function related to the $x$-component of velocity is drawn for different $m(m<0$ shear thinning) values. In Fig.2, the variation of the $K$ related to the $y$-component of the velocity under the same conditions is shown. The decrease in $x$ velocity component values, as shown in Fig. 1 , is related to the decrease in $\mathrm{m}$ values. It is concluded that the boundary layer becomes thicker with decreasing $m$ value. Figure 2 shows the velocity component decrease with the increase in the power-law index $m$. In 
Fig.3, $S$ function and in Fig.4, $K$ function related to the $x$ and $y$ components of the velocities are represented for shear thickening of $m$ values (i.e. $m>0$ ). An increase in $S$ function which is related to the $x$-component of the velocity leads to an increase in $m$ value and the thickness of the boundary layer decreases. In Fig.4, a case opposite to Fig. 3 is observed. In other words, the velocity component decreases as $m$ increases. In Figs 5 and 6 , the effects of the fourth-grade fluid parameter for the positive and negative values of $m$ are shown. In both figures it is seen that the boundary layer thickness decreases with the increase in the value of the parameter. Figures 7 and 8 show the effects of the $c$ parameter. In both figures, $c=1$ that means injection boundary condition, $c=-1$ means suction boundary condition. In the case of $c=1$, it is observed that the boundary layer thickness is greater than that of $c=-1$. In Fig.8, it is seen that the velocity component is higher for $c=1$. For the positive values of $\mathrm{m}$, the change of $c$ parameter is the same as for Figs 7 and 8 .

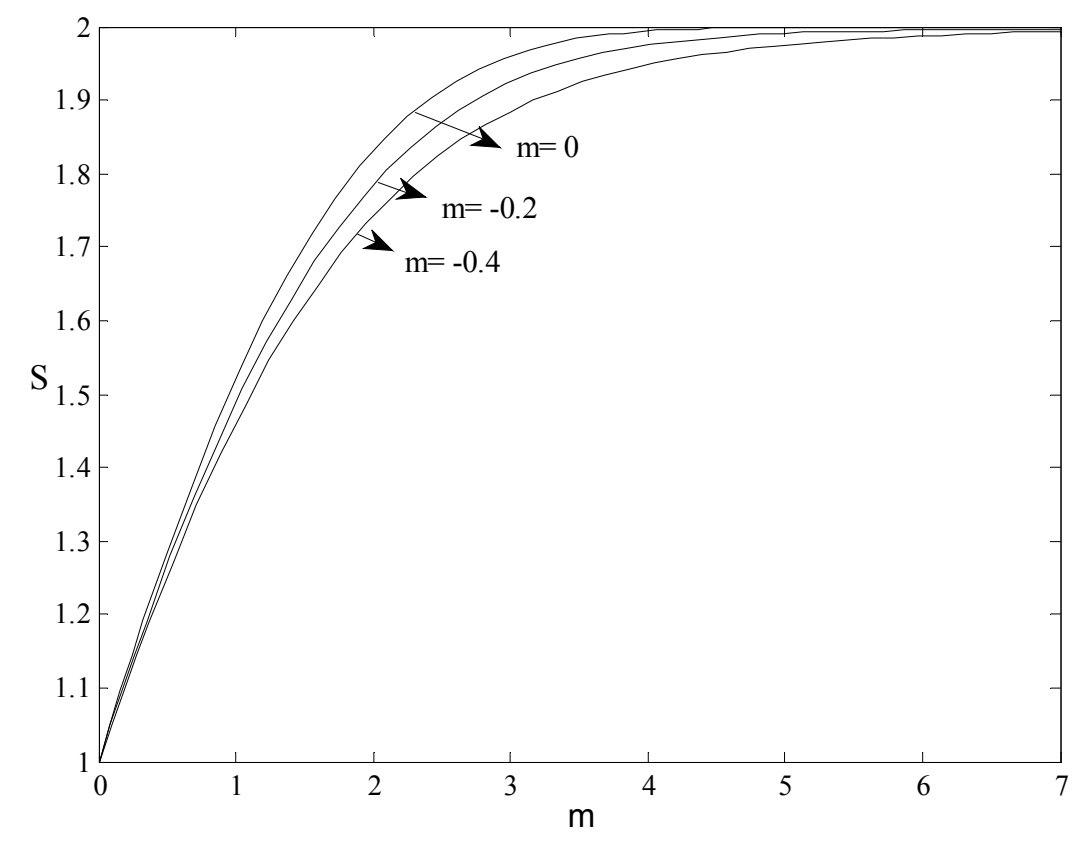

Fig.1. The effect of a parameter $m$ on the $x$-component of velocity for $m<0\left(\gamma=1, n=1, c=1, c_{1}=1, c_{2}=2\right)$.

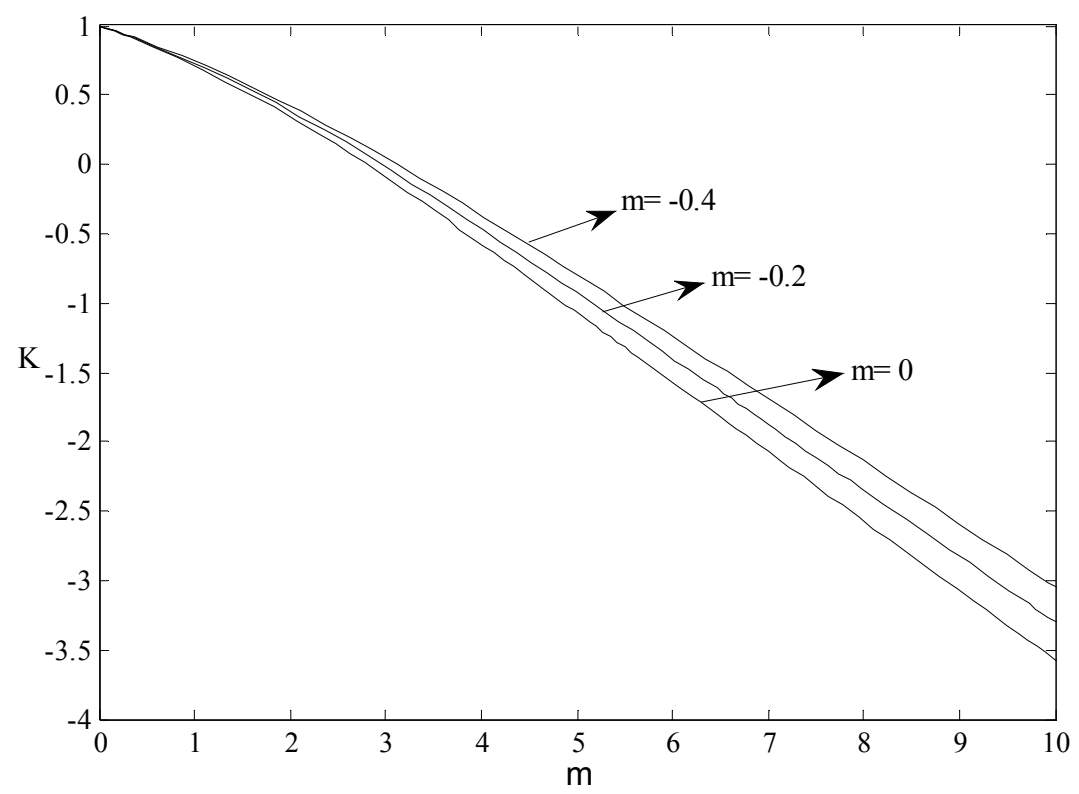

Fig.2. The effect of a parameter $m$ on the $y$-component of velocity for $m<0\left(\gamma=1, n=1, c=1, c_{1}=1, c_{2}=2\right)$. 


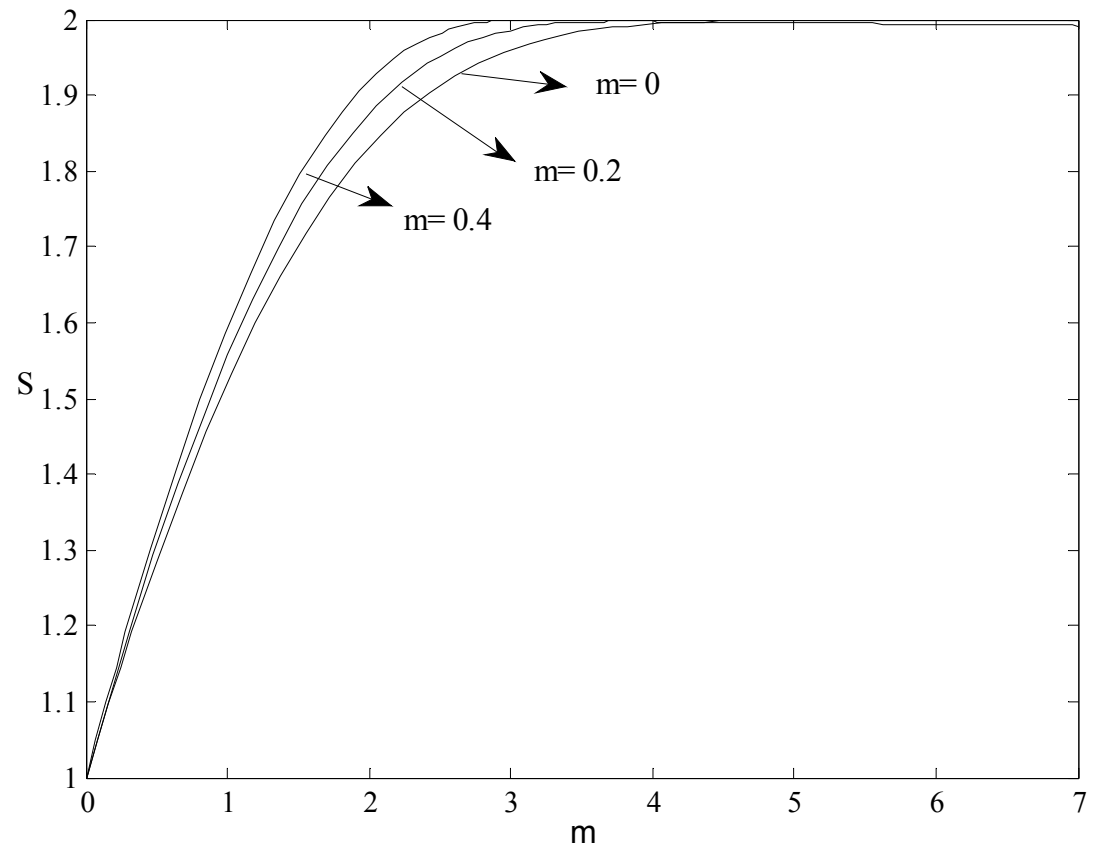

Fig.3. The effect of a parameter $m$ on the $x$-component of velocity for $m>0\left(\gamma=1, n=1, c=1, c_{1}=1, c_{2}=2\right)$.

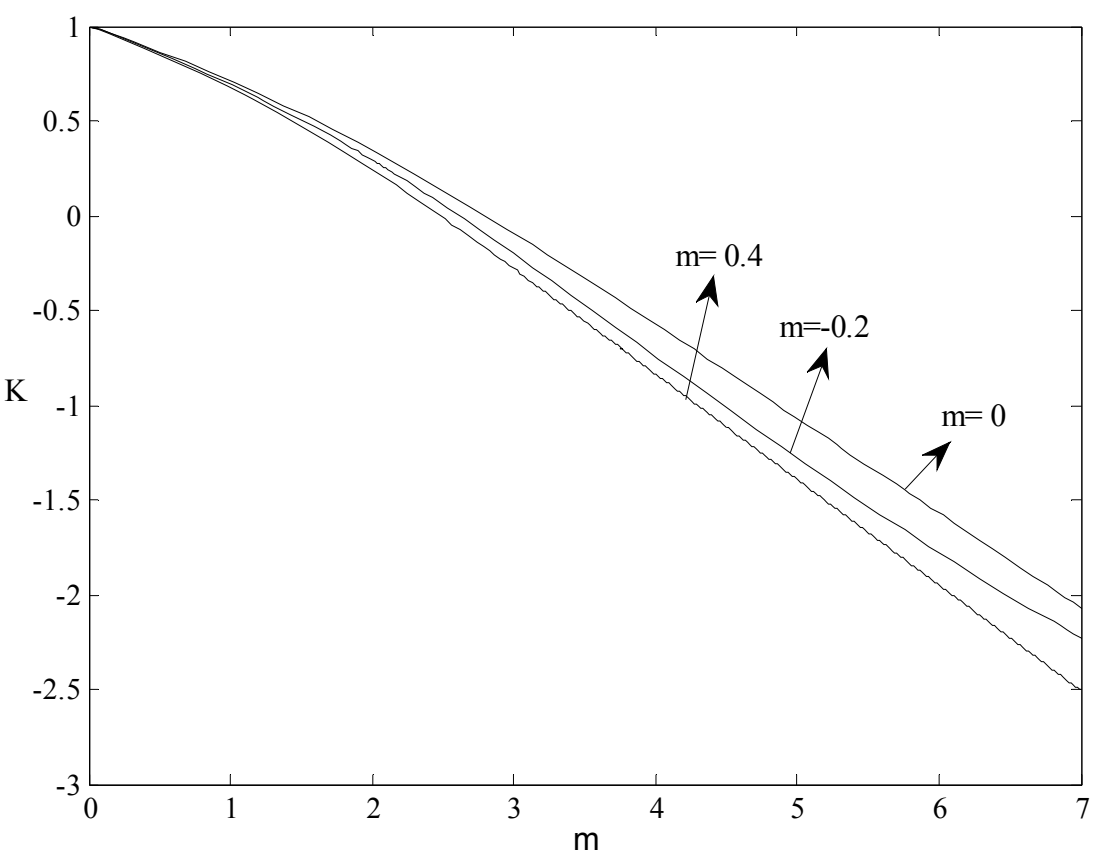

Fig.4. The effect of a parameter $m$ on the $y$-component of velocity for $m>0\left(\gamma=1, n=1, c=1, c_{1}=1, c_{2}=2\right)$. 


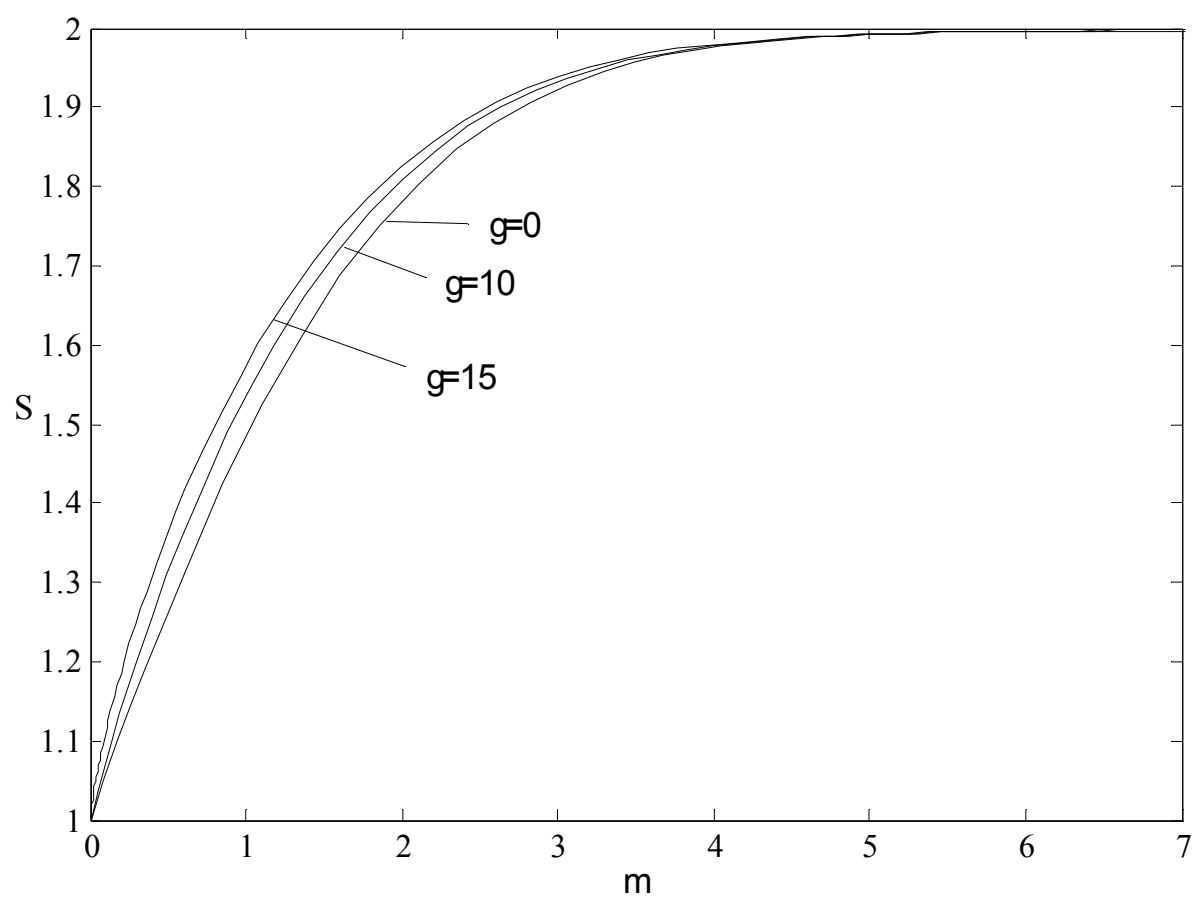

Fig.5. The effect of a fourth grade fluid parameter on the $x$-component of velocity $\left(m=-0.2, n=1, c=1, c_{1}=1, c_{2}=2\right)$.

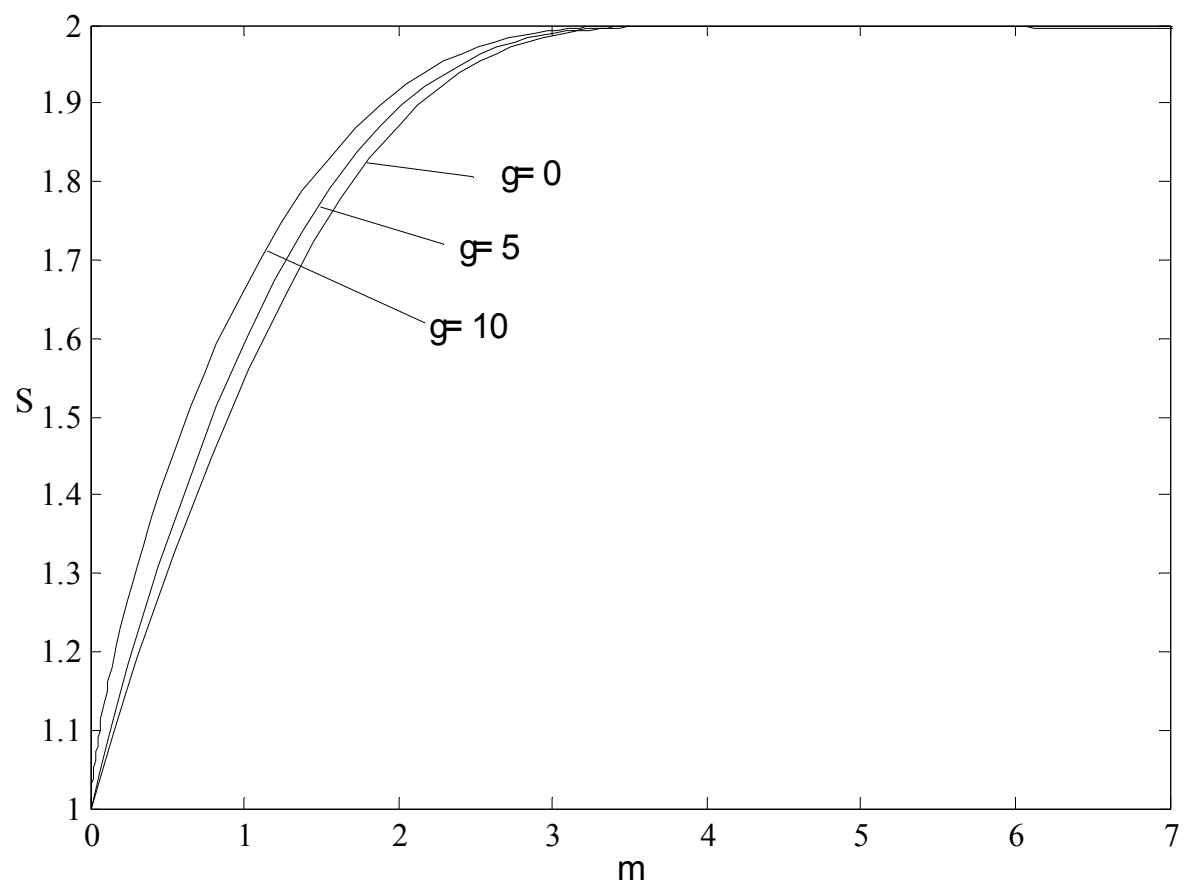

Fig.6. The effect of a fourth grade fluid parameter on the $x$-component of velocity $\left(m=0.2, n=1, c=1, c_{1}=1, c_{2}=2\right)$. 


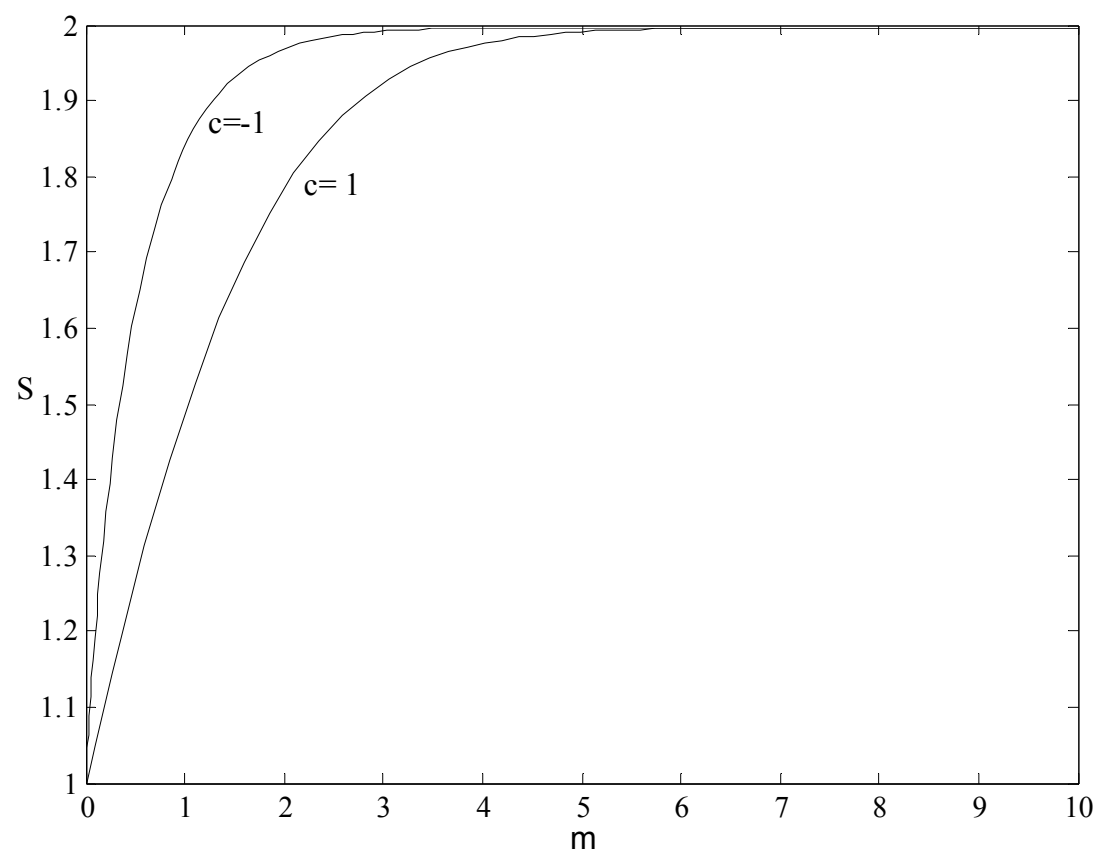

Fig.7. The effect of $c$ parameter on the $x$-component of velocity $\left(\gamma=1, n=1, m=-0.2, c_{1}=1, c_{2}=2\right)$.

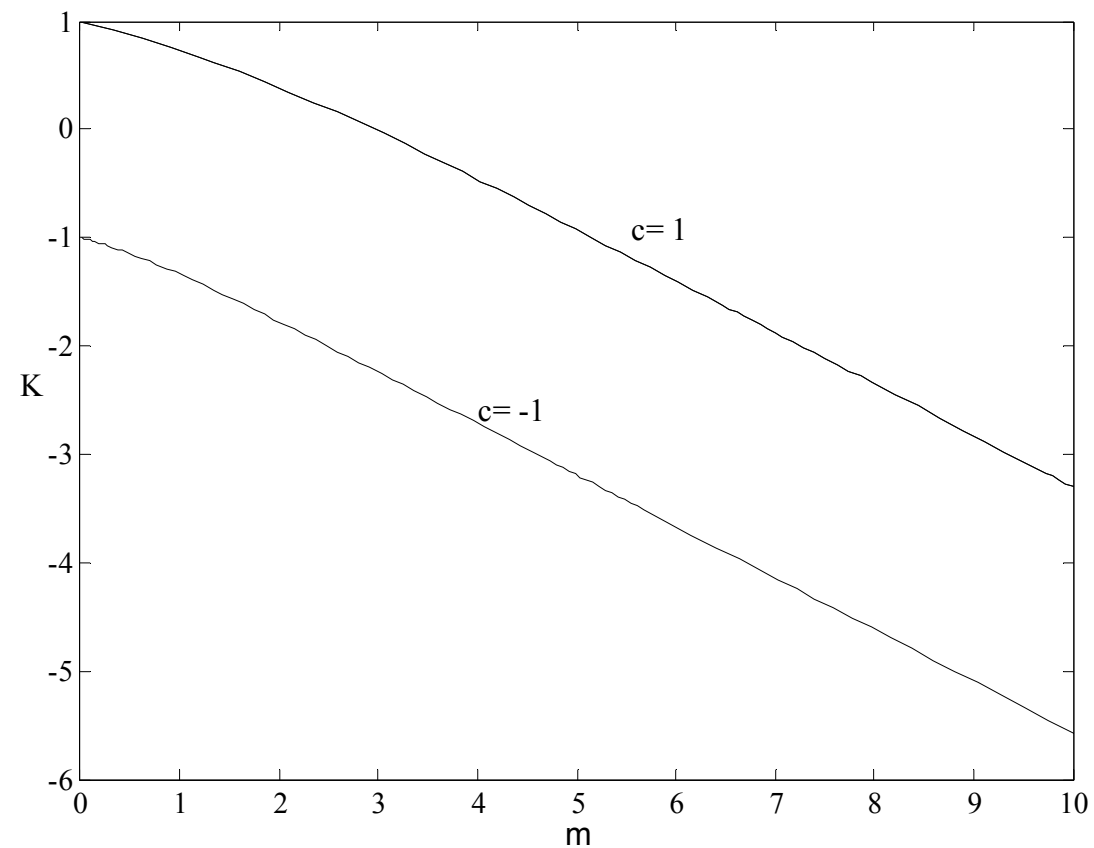

Fig.8. The effect of $c$ parameter on the $y$-component of velocity $\left(\gamma=1, n=1, m=-0.2, c_{1}=1, c_{2}=2\right)$.

Equation (4.6) is numerically integrated, subjected to the boundary conditions (4.7). The functions $P$ and $G$ are plotted in Figs 9-12. The shear thinning and shear thickening cases (i.e., $m<0$ and $m>0$ ) are shown. A decrease in the velocity component value was observed with an increase in $\mathrm{m}$ in both the Figs 10 and 12. Figure 9 is similar to Fig. 11 where the shear thinning and shear thickening velocities approach the edge velocity as an inverse law, whereas in other figures, the velocity change is exponential [4]. 


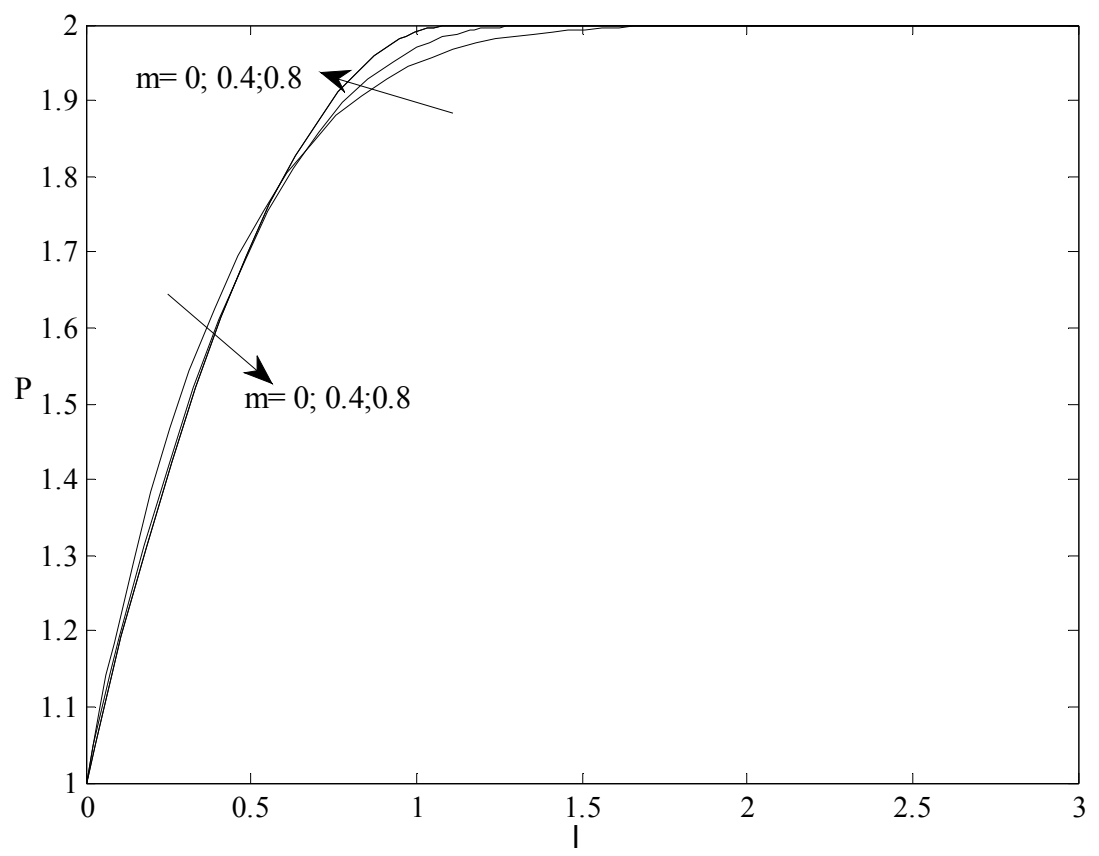

Fig.9. The effect of $m$ parameter on the $x$-component of velocity $\left(\gamma=0.01, c=1, c_{1}=1, c_{2}=2\right)$.

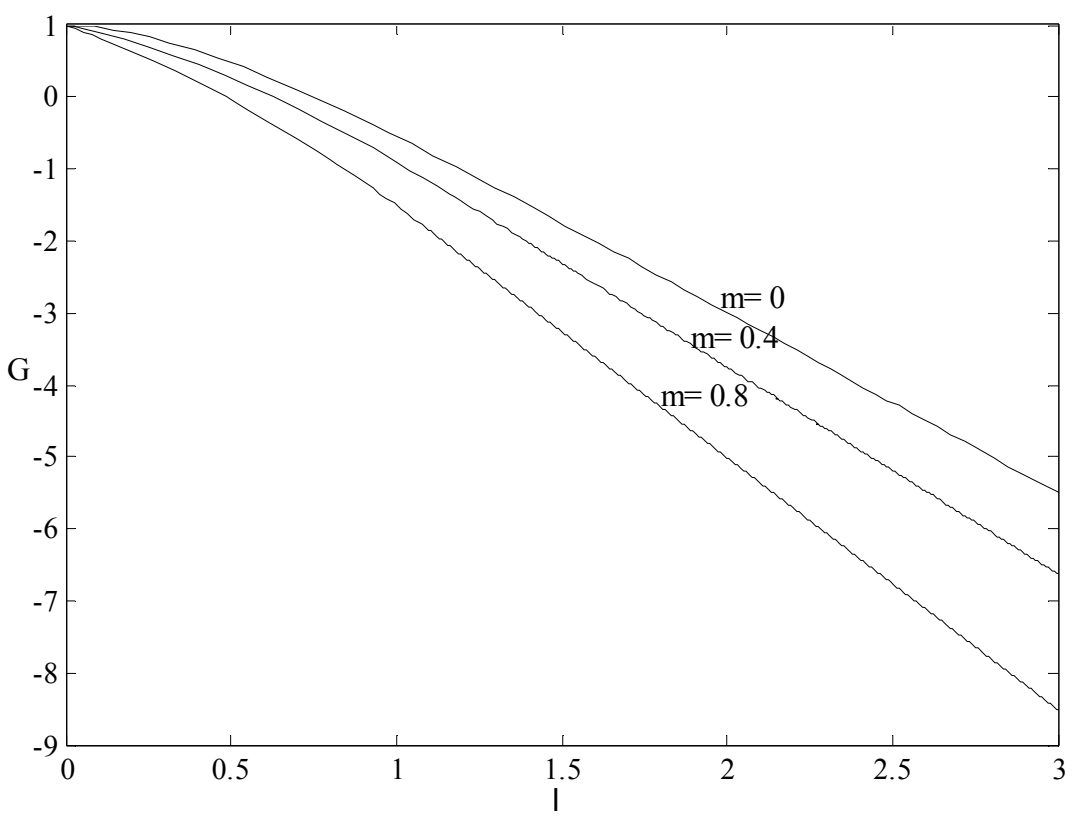

Fig.10. The effect of $m$ parameter on the $y$-component of velocity $\left(\gamma=0.01, c=1, c_{1}=1, c_{2}=2\right)$. 


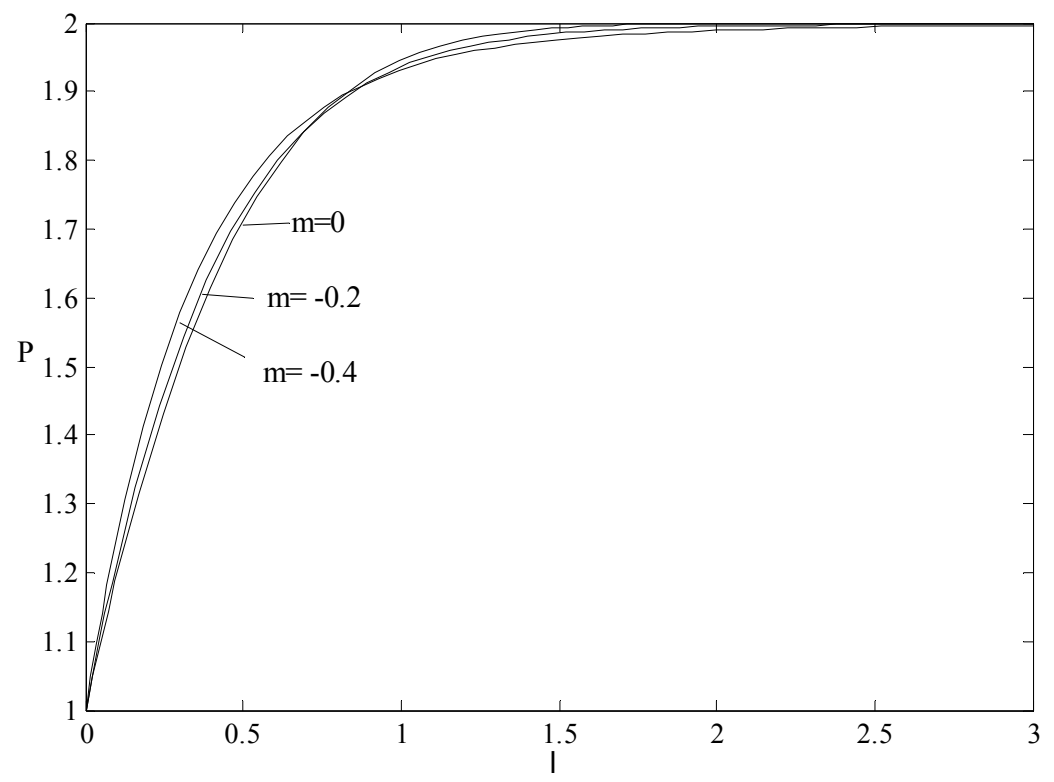

Fig.11. The effect of $m$ parameter on the $x$-component of velocity $\left(\gamma=0.001, c=1, c_{1}=1, c_{2}=2\right)$.

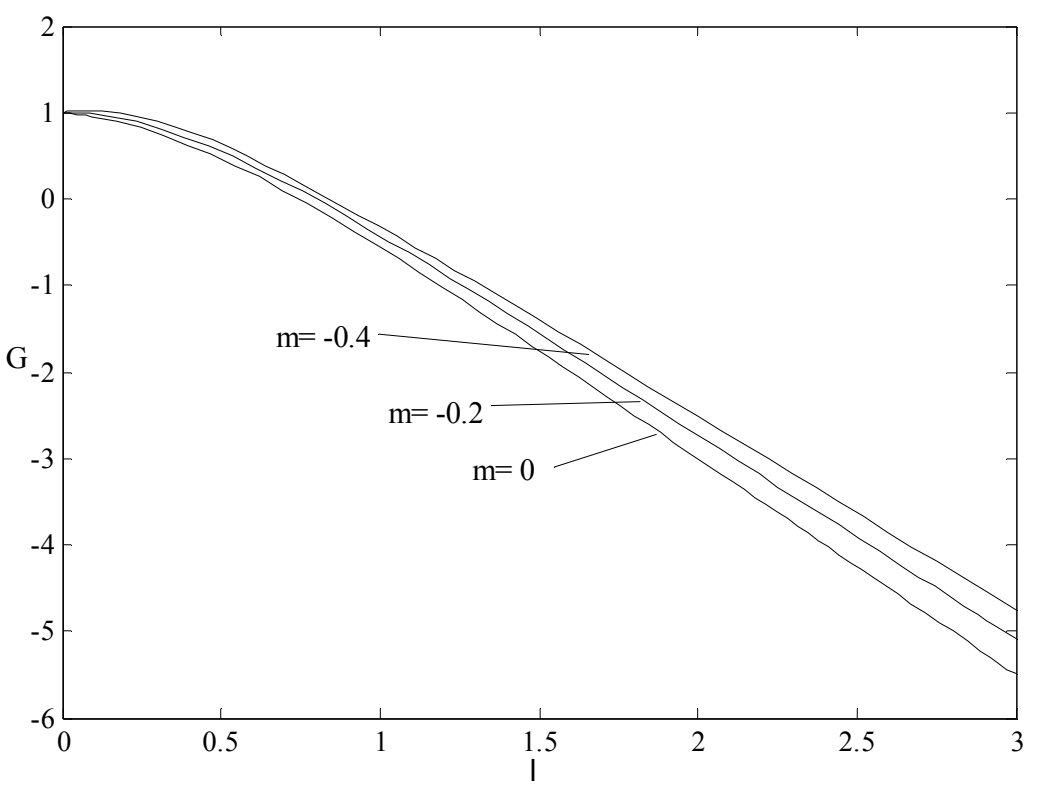

Fig.12. The effect of $m$ parameter on the $y$-component of velocity $\left(\gamma=0.001, c=1, c_{1}=1, c_{2}=2\right)$.

\section{Concluding remarks}

In this study, the time dependent boundary layer flow of a modified power-law fluid of fourth grade on a stretching sheet with suction or injection has been investigated. The flow behaviors of the modified powerlaw fluid of fourth grade were determined by power-law index, fourth grade fluid and suction and injection parameters. Classical single deck unsteady boundary layer equations are derived from the stress tensor. Using scaling and translation transformations, the time dependent partial differential system is transferred to two separate two-independent-variable-partial differential equation system. One of the obtained partial differential equations was reduced to the ordinary differential equation form by using further translation transformation. The other partial differential equation was reduced to the form of ordinary differential 
equation using a combined transformation. Two ordinary differential equations were numerically solved. The effect of the non-Newtonian coefficient and power law index $\mathrm{m}$ on the solutions is investigated. The increase in the non-Newtonian coefficient results in a thinner boundary layer. In one of the equations, the usual solutions for $\mathrm{m}$ parameter were obtained. In the other equation, the qualitative behavior of unsteady boundary layers of the modified fourth grade fluid looks similar to that of a power law fluid for shear thinning and shear thickening cases.

\section{Nomenclature}

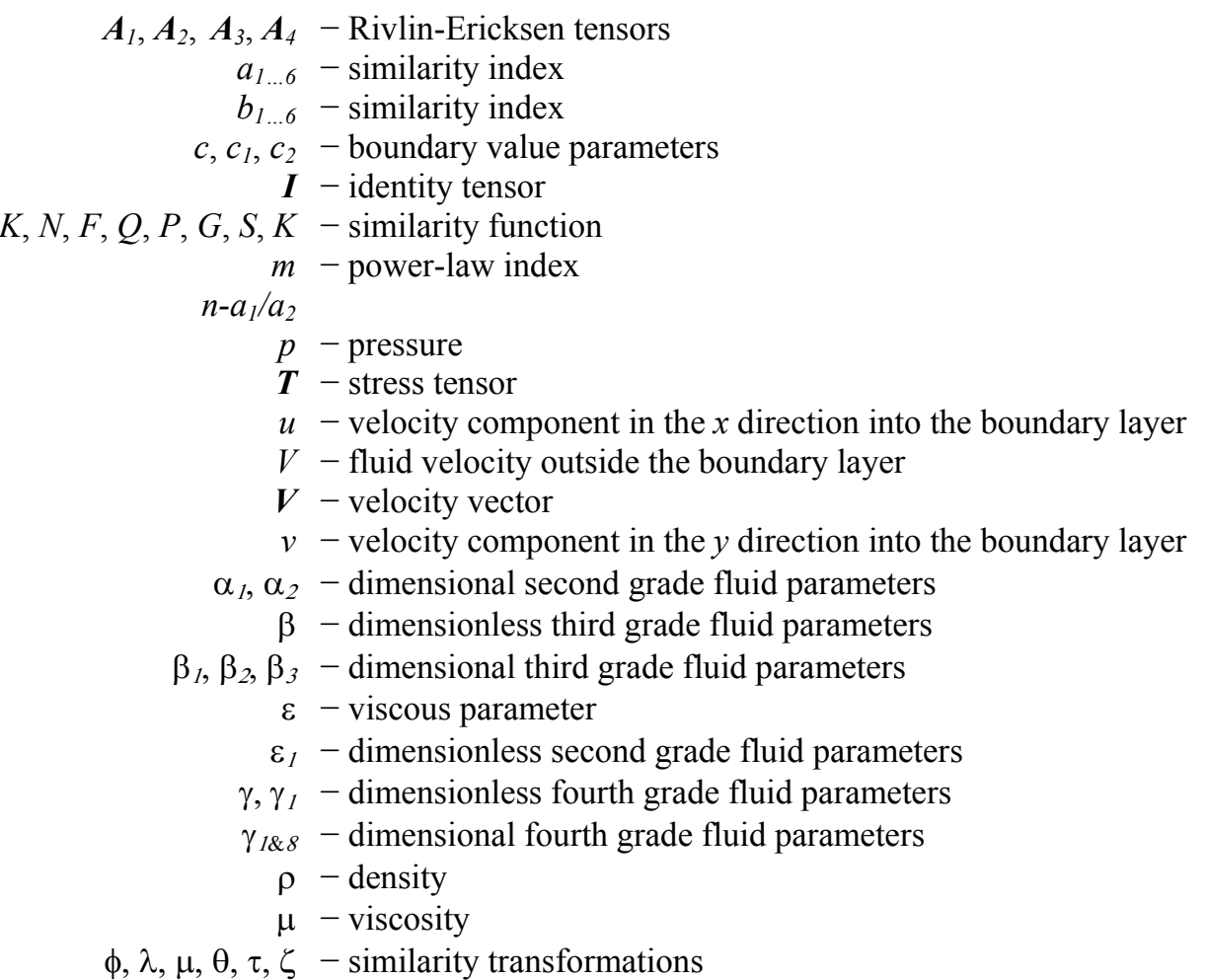

\section{References}

[1] Pakdemirli M. and Suhubi E.S. (1992): Similarity solutions of boundary layer equations for second order fluids. Int. J. Engng Sci., vol.30, pp.611-629.

[2] Pakdemirli M. (1992): The boundary layer equations of third grade fluids. - Int. J. Non-Linear Mech., vol.27, pp.785-793.

[3] Yürüsoy M. and Pakdemirli M. (1997): Symmetry reductions of unsteady three dimensional boundary layers of some non-Newtonian fluids. - Int. J. Engng Sci., vol.35, pp.731-740.

[4] Yürüsoy M. (2006): Unsteady boundary layer flow of power-law fluid on stretching sheet surface. - Int. J. Engng Sci., vol.44, pp.325-332.

[5] Keçebaş A. and Yürüsoy M. (2006): Similarity solutions of unsteady boundary layer equations of a special third grade fluid. - Int. J. Engng Sci., vol.44, pp.721-729.

[6] Pakdemirli M., Aksoy Y., Yürüsoy M. and Khalique C.M. (2008): Symmetries of boundary layer equations of power-law fluids of second grade. - Acta Mech Sin., vol.24, pp.661-670.

[7] Abbasbandy S., Yürüsoy M. and Pakdemirli M. (2008): The analysis approach of boundary layer equations of power-law fluids of second grade. - Z. Naturforsch., vol.63a, pp.564-570. 
[8] Pakdemirli M., Hayat T., Yürüsoy M., Abbasbandy S. and Asghar S. (2011): Perturbation analysis of a modified second grade fluid over a porous plate. - Nonlinear Analysis: Real World Applications, vol.12, pp.1774-1785.

[9] Khan M., Salahuddin T. and Malik M.Y. (2018): An immediate change in viscosity of Carreau nanofluid due to double stratified medium: application of Fourier's and Fick's laws. - Journal of the Brazilian Society of Mechanical Sciences and Engineering, vol.40, pp.457-.

[10] Khan M., Shahid A., Malik M.Y. and Salahuddin T. (2018): Thermal and concentration diffusion in Jeffery nanofluid flow over an inclined stretching sheet: A generalized Fourier's and Fick's perspective. - Journal of Molecular Liquids, vol.251, pp.7-14.

[11] Khan M., Shahid A., Salahuddin T., Malik M.Y. and Mushtaq M. (2018): Heat and mass diffusions for Casson nanofluid flow over a stretching surface with variable viscosity and convective boundary conditions. - Journal of the Brazilian Society of Mechanical Sciences and Engineering, vol.40, pp.533.

[12] Khan M., Malik M.Y., Salahuddin T. and Khan F. (2019):, Generalized diffusion effects on Maxwell nanofuid stagnation point flow over a stretching sheet with slip conditions and chemical reaction. - Journal of the Brazilian Society of Mechanical Sciences and Engineering, vol.41, pp.138.

[13] Khan M., Salahuddin T., Tanveer A., Malik M.Y. and Hussain A. (2018): Change in internal energy of thermal diffusion stagnation point Maxwell nanofluid flow along with solar radiation and thermal conductivity. - Chinese Journal of Chemical Engineering, vol.27, pp.2352-2358.

[14] Yürüsoy M. and Pakdemirli M. (1996): Group-theoretic approach to unsteady boundary-layer equations of some non-Newtonian fluids. - Modern Group Analysis VI, Johennesburg, South Africa.

[15] Ibragimov N.H. (1994): CRC Handbook of lie Group analysis of Differential Equations. - Volume 1, CRC Press, Boca Raton.

[16] Bluman G.W. and Kumei S. (1989): Symmetries and Differential Equations. - New York: Springer-Verlag.

Received: January 9, 2020

Revised: March 21, 2020 